\title{
Interactive comment on "A new method to assess mesoscale contributions to meridional heat transport in the North Atlantic Ocean" by Andrew Delman and Tong Lee
}

\section{Anonymous Referee \#1}

Received and published: 30 April 2020

Review of "A new method to assess mesoscale contributions to meridional heat transport in the North Atlantic Ocean" by Delman and Lee

This manuscript presents interesting results regarding the origin of meridional heat transport fluctuations in the North Atlantic. It is well written and to the point. I recommend publication with minor revisions.

Major comments: 1.The decomposition used by the authors, although mathematically correct, might not represent the effects of the "mesoscale" better than the other methods cited in the introduction. What I have in mind is not so much spatial vs temporal scales but the fact that "mesoscale" fluctuations drive mean circulations which them- 
selves carry heat. These two effects tend to compensate each other so the real effect of the "mesoscale" remain unclear (see for example the Transformed Eulerian Mean framework and its use in discussing tracer transports in the atmosphere-usually some dissipation or diabatic effects are needed to make the compensation imperfect). The new decomposition, although clearly of interest, does not shed light on this and this should be mentioned somewhere in the text. Another way to state this is that it is not clear if the heat transport captured by the author really is a heat transport: a warm blob could be advected poleward at depth (i.e., shielded from air-sea interactions and diabatic effects) for a while and then returned equatorward, still below the mixed layer, without creating a net heat transport in the mean although, in a timeseries, it would show up as an enhanced, then a decreased heat transport. 2.It might be more natural to analyse the heat transport along a mean streamline rather than across $40 \mathrm{~N}$, especially considering the model grid isn't latitude-longitude. This could remove some of the difficulties associated with steady meanders in the model Gulf Stream with scales comparable to those of the "mesoscale" and simplify the physical interpretation of the results (e.g., the cancellations between poleward and equatorward heat transport in Fig. 5a). I understand this isn't trivial to do but if the authors can do it within a reasonable amount of time it would add to the quality of the analysis.

Minor comments: 1 . The length of the integration should be mentioned in the text (I only had a hint of it by looking at the period covered by the timeseries displayed -I couldn't see this information in the model description). 2.line 290: "upgradient heat transport" needs to be stated with caution. You might need to remove the rotational component of the heat transport vector first. 3.Abstract, line 5 ". . . that are more fundamental to the physics of ocean eddies". I am not sure the spatial scales are more fundamental than the temporal scales so maybe this could be removed.

Interactive comment 